\title{
Superballistic wavepacket spreading in double kicked rotors ${ }^{\dagger}$
}

\author{
Ping Fang, and Jiao Wang* \\ Department of Physics and Institute of Theoretical Physics and Astrophysics, Xiamen University, Xiamen 361005, China
}

Received February 23, 2016; accepted March 21, 2016; published online May 16, 2016

\begin{abstract}
We investigate possible ways in which a quantum wavepacket spreads. We show that in a general class of double kicked rotor system, a wavepacket may undergo superballistic spreading; i.e., its variance increases as the cubic of time. The conditions for the observed superballistic spreading and two related characteristic time scales are studied. Our results suggest that the symmetry of the studied model and whether it is a Kolmogorov-Arnold-Moser system are crucial to its wavepacket spreading behavior. Our study also sheds new light on the exponential wavepacket spreading phenomenon previously observed in the double kicked rotor system.
\end{abstract}

quantum chaos, quantum transport, matter waves, semiclassical methods

PACS number(s): 05.45.Mt, 05.60.Gg, 03.75.-b

Citation: $\quad$ P. Fang, and J. Wang, Superballistic wavepacket spreading in double kicked rotors, Sci. China-Phys. Mech. Astron. 59, 680011 (2016), doi: $10.1007 / \mathrm{s} 11433-016-0076-\mathrm{y}$

\section{Introduction}

In a quantum system, a wavepacket usually spreads following a power law of time, i.e., its variance increases in time as $\sim t^{\gamma}$, with $\gamma$ being a constant and $0 \leqslant \gamma \leqslant 2$. For $\gamma=0$, the wavepacket will be localized, while for $\gamma=2$, the wavepacket will spread ballistically. The case of $\gamma=1$ is referred to as "normal diffusion", in contrast to two "anomalous diffusion" cases, i.e., subdiffusion for $0<\gamma<1$ and superdiffusion for $1<\gamma<2$. It is found that under certain conditions the exponent $\gamma$ can be related to the fractal dimension of the system's spectrum [1-3]. For example, for the two special cases of localization $(\gamma=0)$ and ballistic spreading $(\gamma=2)$, the spectrum is discrete and absolutely continuous, respectively.

In recent years, investigations of the possible ways in which a quantum wavepacket spreads have led to some important findings. An interesting example is that [4], if a segment of a one-dimensional (1D) homogenous lattice is replaced by a segment of disordered structure, then a

*Corresponding author (email: phywangj@xmu.edu.cn)

$\nmid$ Recommended by Hong Zhao (Associate Editor) wavepacket that initially resides on the implanted segment may spread "superballistically" with $2<\gamma \leqslant 3$, until a certain characteristic time $T$ that depends on the length of the implanted segment: The longer the latter is, the longer $T$ is. When the time exceeds $T$, the wavepacket tends to converge asymptotically to the ballistic spreading. In a more recent study [5], it was found that for a 1D tight-binding lattice without on-site potential, if one implants a segment of lattice with on-site potential, then a wavepacket initially prepared on the latter may not only spread superballistically, but also hyperdiffusively, i.e., $\gamma$ can be as large as $3<\gamma<5$.

More interestingly, power laws are not the only ways in which a wavepacket spreads. It has been found that a wavepacket can spread in time even exponentially [6]. Similar to the two cases $[4,5]$ mentioned above, the time for which the exponential spreading lasts depends on the system's parameters, which is finite but in principle can be infinite as the system's parameters are tuned. This finding unveils a new type of quantum motion.

The model system in which the exponential spreading was found is a variant of the quantum kicked rotor (QKR) [7]. Despite its seeming simplicity in constructure, the QKR exhibits 
very rich dynamics and has played a central role in quantum chaos studies. Up to now a wide spectrum of wavepacket spreading ways, from power laws with $0 \leqslant \gamma \leqslant 2$ to exponential laws, has been found in the QKR as well as its variants, but superballistic and hyperdiffusive spreading for $\gamma \geqslant 3$ has not been reported yet.

In this work we show that the superballistic spreading with $\gamma=3$ is also possible in a class of kicked rotor systems. This result implies that the superballistic spreading exists in very general systems, not only restricted in the lattices with hybrid structures $[4,5]$. In addition, it suggests a new type of quantum motion in the QKR, evidencing again the dynamics wealth of this paradigmatic quantum chaos model. As various QKR systems have been realized with atom-optics setup [8,9], our result also suggests a possible way to experimental studies of the superballistic spreading.

Based on our understanding of the mechanism for the exponential spreading $[6,10]$, our strategy here is to design variant QKR systems such that the superballistic diffusion can happen in the pseudoclassical limit [11]. Then we show and study the superballistic wavepacket spreading in the original systems, in particular its conditions and characteristics. This method involves the concepts of quantum resonance [12], quantum antiresonance $[12,13]$, pseudoclassical limit theory [11], Kolmogorov-Arnold-Moser (KAM) systems [14], etc. For recent progress in revealing the general wave propagation properties in quantum mechanics and the roles nonlinearities may play in matter wavepacket dynamics, we refer the reader to refs. [15-17]. In the following we will first describe the models to be focused on in sect. 2, then show their quantum wavepacket spreading in sect. 3 and discuss their pseudoclassical systems, the mechanism and the rate of the observed superballistic spreading in sect. 4. Two characteristic time scales will be analyzed in sect. 5. Finally, we will make extended discussions and conclude in sect. 6.

\section{Models}

The 1D kicked rotor system [18] is composed of a point particle confined to move on a circle. The motion of the particle is subjected to a series of sudden kicks imposed periodically, otherwise its motion on the circle is free. Assuming that the inertial moment of the particle and the radius of the circle are unitary, the Hamiltonian of the system is

$\boldsymbol{H}=\frac{p^{2}}{2}+K V(\theta) \sum_{n \in \mathbb{Z}} \delta(t-n \tau)$.

Here $p$ and $\theta \in[-\pi, \pi)$ are the angular momentum and the angular position of the particle, respectively, $V(\theta)$ is the external field of period $2 \pi$, i.e., $V(\theta)=V(\theta+2 \pi)$, and $K$ is a parameter that controls the amplitude of the kicks turned on instantaneously at multiples of $\tau$. In the standard kicked rotor model the potential is $V_{\mathrm{S}}(\theta)=\cos (\theta)$, which is both nondegenerate and sufficiently smooth. Hence the classical standard kicked rotor is a KAM system [14], so that for $K \tau<\kappa_{\mathrm{C}}$ (where $\kappa_{\mathrm{C}} \approx 1$ is a critical value) the motion of the system in the angular momentum space is confined by the Cantori and is localized, but for $K \tau>\kappa_{\mathrm{C}}$ the motion becomes unbounded due to the breaking of all the Cantori, and on average its energy increases linearly in time [18], i.e, $E(t)=p^{2} / 2 \sim K^{2} t$. In sharp contrast, the motion of the quantum standard kicked rotor depends on wether $\hbar \tau$ is an irrational multiple of $\pi$ (here $\hbar$ is the effective Planck constant of the system). If yes, then the energy of the system will eventually saturate, known as the dynamical localization $[7,18]$. Otherwise the so-called quantum resonance occurs, i.e., the energy increases quadratically in time, unless $\hbar \tau$ is an odd integer multiple of $2 \pi$, at which the state of the system goes back to itself after every two kicks and the energy keeps to oscillate periodically. This special case is known as the quantum antiresonance $[12,13]$.

An important variant of the kicked rotor is the double kicked rotor which was first proposed by Li et al. [19] to study the chaos controlling problem in Hamiltonian system. In this model, during a time of $\tau$ the rotor is kicked twice separated by a time interval $\Delta \tau<\tau$. The Hamiltonian is

$\boldsymbol{H}=\frac{p^{2}}{2}+K V(\theta) \sum_{n \in \mathbb{Z}} \delta(t-n \tau)+K V(\theta) \sum_{n \in \mathbb{Z}} \delta(t-n \tau+\Delta \tau)$

Note that the two kicks can be different in general, but in this work we assume they are the same. Quantum mechanically, if we denote the state of the system at the time just before $t=n \tau$ as $|\psi(n)\rangle$, and after a time $\tau$ it evolves into $|\psi(n+1)\rangle$, then the latter can be obtained from the former as $|\psi(n+1)\rangle=U|\psi(n)\rangle$, with the evolution operator

$U=\mathrm{e}^{-\mathrm{i}(\tau-\Delta \tau) \frac{p^{2}}{2 \hbar}} \mathrm{e}^{-\mathrm{i} \frac{K}{\hbar} V(\theta)} \mathrm{e}^{-\mathrm{i} \Delta \tau \frac{p^{2}}{2 \hbar}} \mathrm{e}^{-\mathrm{i} \frac{K}{\hbar} V(\theta)}$.

The second and the forth factors result from kicks and the first and the third term represent the free motion between kicks. In general, the quantum double kicked rotor (QDKR) with potential $V_{\mathrm{S}}$ exhibits dynamical localization: The wavepacket eventually displays exponentially decaying tails in the angular momentum space and the localization length depends on $\hbar$ in a power law with a fractional number exponent [20,21]. The most striking phenomenon of the QDKR appear when the main quantum resonance condition, i.e., $\hbar \tau=4 \pi$, is satisfied, under which the evolution operator reduces to the following more symmetric form:

$U=\mathrm{e}^{\mathrm{i} \frac{p^{2}}{2 \hbar}} \mathrm{e}^{-\mathrm{i} \frac{K}{\hbar} V(\theta)} \mathrm{e}^{-\mathrm{i} \frac{p^{2}}{2 \hbar}} \mathrm{e}^{-\mathrm{i} \frac{K}{\hbar} V(\theta)}$.

Without loss of generality we set $\Delta \tau=1$. This is apparent in view of the fact that $\exp \left(-\mathrm{i} \tau \frac{p^{2}}{2 \hbar}\right)|j\rangle=|j\rangle$ for $\hbar \tau=4 \pi$, where $\{|j\rangle\}$ represent the eigenstates of the angular momentum $p$, i.e.,

$p|j\rangle=j \hbar|j\rangle, \quad\langle\theta \mid j\rangle=\frac{1}{\sqrt{2 \pi}} \mathrm{e}^{\mathrm{i} j \theta}, \quad j \in \mathbb{Z}$.

In the following, we will restrict ourselves on the QDKR defined by the evolution operator given by eq. (4). It has been found that when potential $V_{\mathrm{S}}$ is taken, this system has the 
same spectrum as the kicked Harper model due to the additional symmetry $[22,23]$; Moreover, if $\hbar$ satisfies further the condition $\hbar \approx 2 \pi M / N$, where $M$ and $N$ are odd coprime integers ${ }^{1)}$, a quantum wavepacket that can be readily prepared initially may spread in time exponentially $[6,10]$. As we will show in the following, the exponential spreading is possible thanks to two properties of $V_{\mathrm{S}}$, i.e., its KAM nature and the symmetry of $V_{\mathrm{S}}(\theta)=-V_{\mathrm{S}}(\theta+\pi)$. This potential also has the reflecting symmetry of $V_{\mathrm{S}}(\theta)=V_{\mathrm{S}}(-\theta)$ but this symmetry is irrelevant to the exponential spreading. In order to show this and to explore the superballistic spreading, in this work we consider the QDKR (defined by eq. (4)) with potentials of non-KAM nature and various symmetries. For simulations, we consider three linear piecewise potential functions, denoted as $V_{\mathrm{A}}, V_{\mathrm{B}}$, and $V_{\mathrm{C}}$, that are schematically plotted in Figure 1. All of them are non-analytic and therefore non-KAM. In particular, $V_{\mathrm{A}}$ has two symmetries as $V_{\mathrm{S}}$ does, i.e., $V_{\mathrm{A}}(\theta)=-V_{\mathrm{A}}(\theta+\pi)$ and $V_{\mathrm{A}}(\theta)=V_{\mathrm{A}}(-\theta)$, but $V_{\mathrm{B}}$ and $V_{\mathrm{C}}$ have only the former and the latter, respectively. Our aim is to compare the wavepacket dynamics of the QDKR for these potentials to figure out the key factors for the superballistic and exponential spreading. Our study has clarified that the details of the potential are irrelevant, but the simulation results presented in the following are for the three concrete potential functions with common turning points of $(\theta, p)=(-\pi,-1)$ and $(0,1)$, and extra turning points of $(\theta, p)=(-\pi / 2, g)$ and $(\pi / 2,-g)$ for $V_{\mathrm{B}}$ but $(\theta, p)=(-\pi / 2, g)$ and $(\pi / 2, g)$ instead for $V_{\mathrm{C}}$. Here $g$ is a parameter that we assume to be $g=0.5$; if $g=0$ then both $V_{\mathrm{B}}$ and $V_{\mathrm{C}}$ reduce to $V_{\mathrm{A}}$. Throughout the paper the kicking strength parameter is fixed to be $K=5$ at which the classical limit of the three systems are all chaotic.

\section{Quantum superballistic wavepacket spread- ing}

For a pure state of the QDKR, the kinetic energy of the system $E(t)=\left\langle\psi(t)\left|\frac{p^{2}}{2}\right| \psi(t)\right\rangle$ also represents the variance of the wavepacket in the angular momentum space. We therefore investigate the time dependence of the kinetic energy of our QDKR models. The initial state is set to be $|\psi(0)\rangle=|0\rangle$, i.e., the eigenstate of $p$ with a zero angular momentum. Numerically, we invoke the fast Fourier transform algorithm to simulate the evolution of the system and to calculate $E(t)$ with the obtained $|\psi(t)\rangle$.

We have found that if $\hbar$ is close to a value of $2 \pi M / N$, where $M$ and $N$ are odd coprime integers, for the QDKR with potential $V_{\mathrm{A}}$ and $V_{\mathrm{B}}$ the wavepacket undergoes the superballistic spreading. This is the main result of this work. Examples for the case of $\hbar \approx 2 \pi$ and $\hbar \approx 2 \pi / 3$ are shown in Figures 2(a), (b) and see one example in sect. 6, respectively. Generally, $E(t)$ displays three stages, and the two characteristic time scales are denoted as $t_{\mathrm{c}}$ and $t_{\mathrm{s}}$ (indicated by the arrows in Figures 2(a) and (b)), respectively. At the first stage for $t<t_{\mathrm{c}}$, the wavepacket spreads ballistically, $E(t) \sim t^{2}$; At the second stage for $t_{\mathrm{c}}<t<t_{\mathrm{s}}$, it spreads superballistically, $E(t) \sim t^{3}$. Finally, for $t>t_{\mathrm{s}}$, the superballistic spreading is suppressed and $E(t)$ begins to oscillate around a certain value denoted as $E_{\mathrm{s}}$. For potential $V_{\mathrm{C}}$ the intermediate superballistic stage is missing; there are only two stages left: the ballistic stage is followed by the oscillating one.
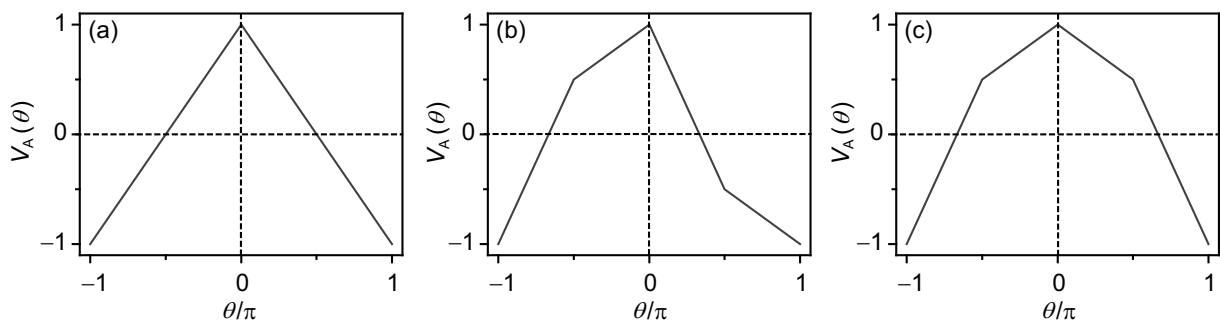

Figure 1 Three linear piecewise potential functions of the $1 \mathrm{D}$ double kicked rotor studied in this work. Potential $V_{\mathrm{B}}$ and $V_{\mathrm{C}}$ have the symmetry of $V_{\mathrm{B}}(\theta)=-V_{\mathrm{B}}(\theta+\pi)$ and $V_{\mathrm{C}}(\theta)=V_{\mathrm{C}}(-\theta)$, respectively, while potential $V_{\mathrm{A}}$ have both.
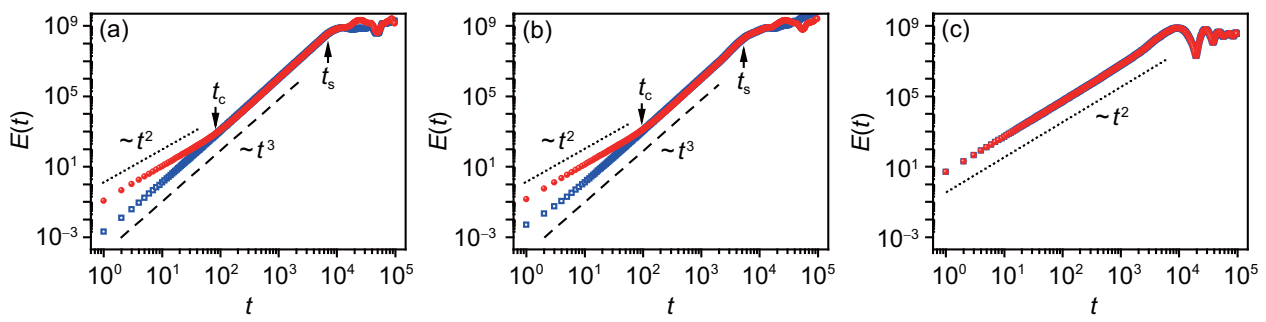

Figure 2 (Color online) Time dependence of the energy of both the QDKR (red solid bullets) and its pseudoclassical system (blue open squares) for three potential functions, (a) $V_{\mathrm{A}}$; (b) $V_{\mathrm{B}}$; and (c) $V_{\mathrm{C}}$, respectively. In each panel, the time scaling $\sim t^{2}$ and $\sim t^{3}$ are indicated by the dotted and the dashed line, respectively, for reference. In all the cases $\hbar=2 \pi+\tilde{\hbar}$ and $\tilde{\hbar}=10^{-3}$.

1) As $\hbar \tau=4 \pi, \Delta \tau=1$ and $\tau>\Delta \tau$, the condition $\hbar \approx 2 \pi M / N$ can be satisfied by adjusting the parameter $\tau$ so that $\tau \approx 2 N / M>1$. This implies that $2 N>M$, which is assumed in the following. 
Denote the deviation of $\hbar$ from $2 \pi M / N$ as $\tilde{\hbar}$, i.e., $\hbar=$ $2 \pi M / N+\tilde{\hbar}$, our numerical analysis has established the following scaling ${ }^{2)}$ :

$t_{\mathrm{c}} \sim 1 / \sqrt{\tilde{\hbar}}, t_{\mathrm{s}} \sim 1 / \tilde{\hbar}, \quad E_{\mathrm{s}} \sim 1 / \tilde{\hbar}^{2}$.

It implies that though the superballistic stage is finite, it can be arbitrarily long by decreasing the parameter $\tilde{\hbar}$. In the following sections we will discuss further these relations and why for potential $V_{C}$ the superballistic spreading does not occur.

\section{Pseudoclassical limit at $\hbar \approx 2 \pi$}

For both the QKR and the DQKR with potential $V_{\mathrm{S}}$, the special case of $\hbar=2 \pi+\tilde{\hbar}(|\tilde{\hbar}| \ll 1)$ is of special interest because it allows one to analyze the system by a classical method $[6,11]$. This is known as the pseudoclassical limit theory [11], which maps the system onto a virtual classical system by assuming $\tilde{\hbar}$ as a virtual Planck constant and taking the virtual classical limit of $\tilde{\hbar} \rightarrow 0$. We find that the pseudoclassical limit theory is also valid in dealing with our DQKR models of non-KAM potentials. In this section we use this method to explore the mechanism and the properties of the superballistic spreading. Let

$\tilde{\theta}=\theta, \quad \tilde{p}=p \tilde{\hbar} / \hbar, \quad \tilde{K}=K \tilde{\hbar} / \hbar$,

the operator $U$ defined by eq. (4) can be rewritten as:

$\tilde{U}=\mathrm{e}^{\frac{\mathrm{i}}{\tilde{\hbar}}\left(\frac{\tilde{p}^{2}}{2}+\pi \tilde{p}\right)} \mathrm{e}^{-\mathrm{i} \frac{\tilde{K}}{\hbar} V(\tilde{\theta})} \mathrm{e}^{-\frac{\mathrm{i}}{\hbar}\left(\frac{\tilde{p}^{2}}{2}+\pi \tilde{p}\right)} \mathrm{e}$

This implies a rotor that is described by the conjugate pair $(\tilde{\theta}, \tilde{p})$ and is subjected to the external field $\tilde{K} V(\tilde{\theta})$. In the pseudoclassical limit of $\tilde{\hbar} \rightarrow 0$, this rotor undergoes a free rotation with the angular velocity $\mathrm{d} \tilde{\theta} / \mathrm{d} t=\tilde{p}+\pi$ or $-(\tilde{p}+\pi)$ alternatively between two consequential kicks, and the corresponding classical motion within a single step is given by the $\operatorname{map} M:\left(\tilde{\theta}_{n}, \tilde{p}_{n}\right) \rightarrow\left(\tilde{\theta}_{n+1}, \tilde{p}_{n+1}\right)$,

$M:\left\{\begin{array}{l}\rho=\tilde{p}_{n}+\tilde{K} f\left(\tilde{\theta}_{n}\right), \\ o=\tilde{\theta}_{n}+\rho+\pi, \\ \tilde{p}_{n+1}=\rho+\tilde{K} f(o), \\ \tilde{\theta}_{n+1}=o-\tilde{p}_{n+1}+\pi,\end{array}\right.$

where $f(\theta) \equiv-\mathrm{d} V(\theta) / \mathrm{d} \theta$ ( $\rho$ and $o$ are two intermediate variables). This defines the classical kicked rotor system in the pseudoclassical limit, which, in the following we refer to as the pseudoclassical system of the QDKR. We are interested in the "energy" of this pseudoclassical system $\tilde{E} \equiv\left\langle\tilde{p}^{2} / 2\right\rangle$, where the average is taken over an ensemble of initial conditions. In order to compare the results with those of the QDKR, we assume the rescaled energy, denoted by $E(t)$ as well without confusion, that $E(t) \equiv \tilde{E}(t) \hbar^{2} / \tilde{\hbar}^{2}$. We also assume the initial conditions $\left(\tilde{\theta}_{0}, \tilde{p}_{0}\right)$ that match the initial quantum state $|0\rangle$; i.e., $\tilde{p}_{0}=0$ and $\tilde{\theta}_{0}$ distributes uniformly in $[-\pi, \pi)$. In our simulations, an ensemble of $10^{4}$ initial conditions is adopted.

The results of $E(t)$ for the pseudoclassical systems with the three linear piecewise potentials are shown in Figure 2 for $\hbar=2 \pi+\tilde{\hbar}$ and $\tilde{\hbar}=10^{-3}$. It can be seen that for potentials $V_{\mathrm{A}}$ and $V_{\mathrm{B}}, E(t)$ increases with the cubic of time all the way until $t=t_{\mathrm{s}}$. In addition, the agreement between the QDKR systems and their pseudoclassical systems is perfect for $t>t_{\mathrm{c}}$. For potential $V_{\mathrm{C}}$, the pseudoclassical system also undergoes ballistic motion, and the agreement with the QDKR is perfect as well. These results suggest that the pseudoclassical limit theory works well for our models despite of their non-KAM nature, and the ballistic spreading of the QDKR with $V_{\mathrm{A}}$ and $V_{\mathrm{B}}$ is a pure quantum effect.

In order to understand the mechanism of the observed superballistic (for $V_{\mathrm{A}}$ and $V_{\mathrm{B}}$ ) and ballistic (for $V_{\mathrm{C}}$ ) motions, we plot the phase portraits of the three pseudoclassical systems in Figure 3. It shows that for $V_{\mathrm{A}}$ and $V_{\mathrm{B}}$ there is a common feature that is absent for $V_{C}$ : There is a horizontal phase line along the $\tilde{\theta}$ axis. For $V_{\mathrm{A}}$ (see Figure 3(a)), checking further the motion of the phase points one may find that a phase point on the positive half of $\tilde{\theta}$-axis moves to the right at a constant speed until it reaches $\tilde{\theta}=\pi$, then the point moves up towards the point $(\tilde{\theta}, \tilde{p})=(0,2 \pi)$, also at a constant speed. The motion of the points on the negative half of $\tilde{\theta}$-axis is the same but along the opposite direction. This observation explains why $\tilde{E}(t) \sim t^{3}$. In fact, $\tilde{E}(t) \sim P_{\tilde{p} \neq 0}(t) t^{2}$, where $P_{\tilde{p} \neq 0}(t)$ stands for the potion of phase points in the average ensemble that have left $\tilde{\theta}$-axis at time $t$, which increases as $\sim t$, and $t^{2}$ stands for the contributions of these points to the averaged energy because their momentum increases linearly; i.e., $\tilde{p}(t) \sim t$. This mechanism is the same in spirit as that outlined in ref. [4]. For $V_{\mathrm{B}}$ this explanation still works, despite of the fact that the motion of phase points on $\tilde{\theta}$-axis takes two different constant speeds instead because $\left|\partial V_{\mathrm{B}}(\theta) / \partial \theta\right|$ has two different values. For $V_{\mathrm{C}}$ after one step of evolution all the phase points in the average ensemble will leave $\tilde{\theta}$-axis and begin to move ballistically, hence we have $P_{\tilde{p} \neq 0}(t)=1$ for $t>0$ and $\tilde{E}(t) \sim t^{2}$.

It is important to note that the phase space structure has a period $2 \pi$ in $\tilde{p}$ direction. Consequently, the superballistic (for $V_{\mathrm{A}}$ and $V_{\mathrm{B}}$ ) and the ballistic (for $V_{\mathrm{C}}$ ) diffusion does not last forever. When the phase points reach $|\tilde{p}| \approx 2 \pi$ their motion directions may change, and therefore the energy increasing slows down. For the cases shown in Figure 2, $E_{\mathrm{s}}$ can then be estimated to be $\tilde{E}_{\mathrm{s}} \hbar^{2} / \tilde{\hbar}^{2} \approx 8 \times 10^{8}$, which agrees very well with the simulation results (see Figure 2).

With the help of the classical motion equation given by map $M$, we can further determine the prefactor of the superballistic and the ballistic diffusion. As an example here we consider the case with potential $V_{\mathrm{A}}$, but the calculations can be extended to other two cases straightforwardly. Based on eq. (9), after one step of iteration a given initial condition $\left(\tilde{\theta}_{0}, \tilde{p}_{0}\right)\left(\tilde{p}_{0}=0\right)$ is mapped to

2) We assume $\tilde{\hbar} \geqslant 0$ throughout but $\tilde{\hbar}$ can be negative (see ref. [11]) and all the results presented in this work can be extended to $\tilde{\hbar}<0$ straightforwardly. 

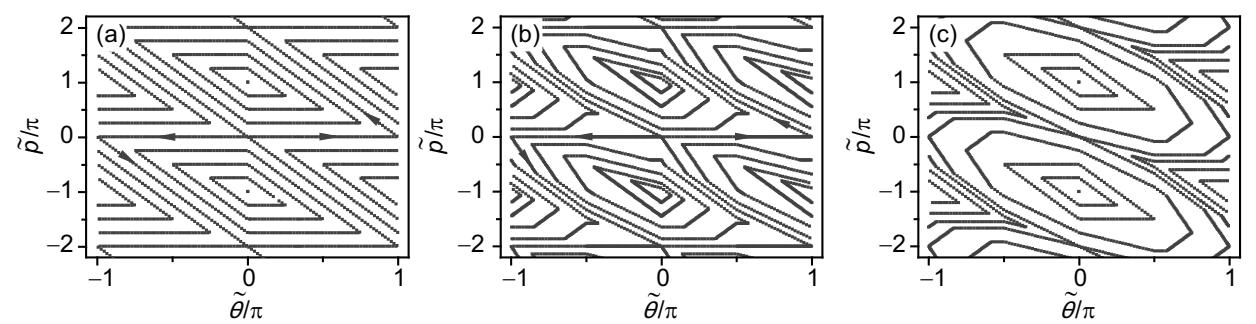

Figure 3 The phase space structure of the system in the pseudoclassical limit of the QDKR with potential given by $V_{\mathrm{A}}$ (a), $V_{\mathrm{B}}$ (b), and $V_{\mathrm{C}}$ (c), respectively. The arrows in (a) and (b) indicate the moving direction of phase points on the negative and positive half of $\tilde{\theta}$-axis. All the parameters are the same as in Figure 2 .

$\left(\tilde{\theta}_{1}, \tilde{p}_{1}\right)= \begin{cases}\left(\tilde{\theta}_{0}-\Delta, 2 \Delta\right), & \text { for } \pi-\Delta \leqslant \tilde{\theta}_{0}<\pi, \\ \left(\tilde{\theta}_{0}+\Delta, 0\right), & \text { for } 0 \leqslant \tilde{\theta}_{0}<\pi-\Delta, \\ \left(\tilde{\theta}_{0}-\Delta, 0\right), & \text { for } \Delta-\pi \leqslant \tilde{\theta}_{0}<0, \\ \left(\tilde{\theta}_{0}+\Delta, 2 \Delta\right), & \text { for }-\pi \leqslant \tilde{\theta}_{0}<\Delta-\pi,\end{cases}$

where $\Delta \equiv 2 \tilde{K} / \pi$. It shows that after one step of iteration, a portion of $\Delta / \pi$ phase points in the initial condition ensemble increases by an amount of $2 \Delta^{2}$ in their energy. Repeating this calculation, one finds that after $n$ steps of iteration, $n$ portions of initial conditions have, respectively, energy $2 \Delta^{2}, 4 \Delta^{2}, \cdots$, $2 n^{2} \Delta^{2}$, before the momentum of the portion with the largest energy $2 n^{2} \Delta^{2}$ reaches $|\tilde{p}| \approx 2 \pi$. It follows that in the time range $t<t_{\mathrm{s}}$ with

$t_{\mathrm{s}} \approx \frac{\pi}{\Delta}=\frac{\pi^{2} \hbar}{2 K \tilde{\hbar}}$

the ensemble averaged energy increases as:

$\tilde{E}(t)=\frac{\Delta^{3}}{3 \pi} t(t+1)(2 t+1) \approx \frac{2 \Delta^{3}}{3 \pi} t^{3}$,

or in terms of $E(t)$, that

$E(t)=\tilde{E}(t) \frac{\hbar^{2}}{\tilde{\hbar}^{2}} \approx \frac{16 K^{3} \tilde{\hbar}}{3 \pi^{4} \hbar} t^{3}$.

These results have been fully corroborated by the simulations.

Now it is in order to explain why for $V_{\mathrm{A}}$ and $V_{\mathrm{B}}$ there is the crucial phase space structure along $\tilde{\theta}$-axis but for $V_{C}$ there is not. For an initial phase point $\left(\tilde{\theta}_{0}, \tilde{p}_{0}\right)$ on $\tilde{\theta}$-axis, i.e., $\tilde{p}_{0}=0$, it can be obtained from eq. (9) that $\tilde{p}_{1}=$ $\tilde{K}\left[f\left(\tilde{\theta}_{0}\right)+f\left(\tilde{\theta}_{0}+\tilde{K} f\left(\tilde{\theta}_{0}\right)+\pi\right)\right]$; Considering that $\tilde{K}=\tilde{\hbar} K / \hbar$ can be much smaller than one due to $\tilde{\hbar} \ll 1$, it implies that in general $\tilde{p}_{1} \approx \tilde{K}\left[f\left(\tilde{\theta}_{0}\right)+f\left(\tilde{\theta}_{0}+\pi\right)\right]$. So after one step of iteration a typical initial phase point will leave $\tilde{\theta}$ axis. However, if the potential has the symmetry $V(\tilde{\theta})=$ $-V(\tilde{\theta}+\pi)$, then $f(\tilde{\theta})$ has the same symmetry and as a result $\tilde{p}_{1}=\tilde{K}\left[f\left(\tilde{\theta}_{0}\right)-f\left(\tilde{\theta}_{0}+\tilde{K} f\left(\tilde{\theta}_{0}\right)\right)\right]=O\left(\tilde{K}^{2}\right)$ if $V$ is smooth enough. Therefore, up to the first order of $\tilde{K}$ we have $\tilde{p}_{1}=0$ (In fact, for $V_{\mathrm{A}}$ and $V_{\mathrm{B}}$, we can prove that $p_{1}=0$ exactly if $\tilde{\theta}_{0}$ and $\tilde{\theta}_{0}+\tilde{K} f\left(\tilde{\theta}_{0}\right)$ are on the same line segment of the potential function). This argument works not only for $V_{\mathrm{A}}$ and $V_{\mathrm{B}}$, but also for other non-KAM potentials (see one example presented in the sect. 6) and KAM potentials (such as $V_{\mathrm{S}}$ ) of the same symmetry. For a KAM system, the difference is that there are hyperbolic fixed points on $\tilde{\theta}$-axis; they make the nearby phase points approach and leave them exponentially and thus induce the exponential wavepacket spreading instead $[6,10]$.

\section{Two characteristic time scales}

Now let us come back to the QDKR models. As their pseudoclassical systems mimic their motions closely, we are able to explore their motions via the latter. In this section we discuss the times $t_{\mathrm{c}}$ and $t_{\mathrm{s}}$ that characterize the quantum superballistic spreading. For the sake of convenience let us first consider the case of potential $V_{\mathrm{A}}$ and $\hbar=2 \pi+\tilde{\hbar}(\tilde{\hbar} \ll 1)$. For this case we have obtained $t_{\mathrm{s}}$ in the pseudoclassical system and found that it applies to the QDKR system equally. However, as the pseudoclassical system does not show the ballistic motion in the initial stage for $t<t_{\mathrm{c}}$, we can not probe the clues of the time $t_{\mathrm{c}}$ from it.

In fact, the initial ballistic spreading of the considered QDKR is a result of the quantum antiresonance. To show this let us assume $\tilde{\hbar}=0$ and $\hbar=2 \pi$ so that $\exp \left( \pm \mathrm{i} \frac{p^{2}}{2 \hbar}\right)|k\rangle=$ $(-1)^{k}|k\rangle$. Taking this into account, we can write the matrix elements of operator $U$ (eq. (4)) as:

$\langle j|U| k\rangle=\frac{(-1)^{j+k}}{2 \pi} \int_{-\pi}^{\pi} \mathrm{d} \theta \mathrm{e}^{\mathrm{i}(k-j) \theta} \mathrm{e}^{-\mathrm{i} \frac{K}{\hbar}[V(\theta)+V(\theta+\pi)]}$.

It follows that for $V_{\mathrm{A}}$, which satisfies $V_{\mathrm{A}}(\theta)=-V_{\mathrm{A}}(\theta+\pi)$, $U$ reduces to the identity operator [13]. We have the same property for $V_{\mathrm{B}}$ but not for $V_{\mathrm{C}}$. Now, if $\tilde{\hbar}$ is slightly changed, we find that locally $U$ remains close to the identity, i.e., for $|j|,|k|<1 / \sqrt{\tilde{\hbar}}$,

$\langle j|U| k\rangle \approx \begin{cases}1-O(\tilde{\hbar}), & \text { for } j=k, \\ O\left(\tilde{\hbar}^{3 / 2}\right), & \text { for } j \neq k .\end{cases}$

This gives $\left\langle j\left|U^{t}\right| k\right\rangle \approx t\langle j|U| k\rangle(j \neq k)$ and

$E(t)=\frac{\hbar^{2}}{2} \sum_{j} j^{2}\left|\left\langle j\left|U^{t}\right| k\right\rangle\right|^{2} \approx D t^{2}$

for the initial state $|0\rangle$ with

$D=\frac{\hbar^{2}}{2} \sum_{j} j^{2}|\langle j|U| 0\rangle|^{2}$. 
This explains why $E(t)$ increases ballistically at the initial stage for the QDKR with potential $V_{\mathrm{A}}$ and $V_{\mathrm{B}}$. Numerically, we can further determine $D$ up to a numerical prefactor, i.e.,

$D \sim K^{2} \sqrt{\tilde{\hbar}}$.

For the QDKR with potential $V_{\mathrm{A}}$, if we identify its superballistic spreading process with that of its pseudoclassical system, then by combining eqs. (13) and (18), we find

$t_{\mathrm{c}} \sim \frac{1}{K \sqrt{\tilde{\hbar}}}$

We put eqs. (19) and (11) into numerical tests and show the results are in Figure 4(b). It can be seen that the simulation results fit them very well. Numerically, $t_{\mathrm{c}}$ and $t_{\mathrm{s}}$ are determined by best fitting $E(t)$ with $\sim t^{2}$ and $\sim t^{3}$ respectively in the ballistic and superballistic stage and extrapolating the two best fitting lines: Their intersection point gives $t_{\mathrm{c}}$ and the time at which $E(t)$ begins to deviate from the extrapolated best fitting line over the superballistic stage gives $t_{\mathrm{s}}$ (see Figure 4(a)).

For other cases in this study where the superballistic spreading is observed, the numerical factors of $t_{\mathrm{c}}$ and $t_{\mathrm{s}} \mathrm{ob}$ tained via this special example are different, but their dependence on $\tilde{\hbar}$, i.e., $t_{\mathrm{c}} \sim 1 / \tilde{\hbar}^{0.5}$ and $t_{\mathrm{s}} \sim 1 / \tilde{\hbar}$, still holds, as confirmed by extensive simulations. Hence the scaling relations given by eq. (6) are expected to be valid generally for the superballistic spreading in non-KAM QDKR systems.

\section{Discussions and summary}

In the last two sections we have mainly studied the special case of $V_{\mathrm{A}}$ and $\hbar=2 \pi+\tilde{\hbar}(\tilde{\hbar} \ll 1)$. In fact the superballistic wavepacket spreading have been found to generally occur in the QDKR with other non-KAM potentials of symmetry $V(\theta)=-V(\theta+\pi)$ and other $\hbar$ values close to $2 \pi M / N$. For example, in Figure 4(c) we present the simulation results of $E(t)$ for potential $V_{\mathrm{A}}$ but at $\hbar \approx 2 \pi / 3$, from which the characteristics of the superballistic spreading similar to the case of $\hbar \approx 2 \pi$ can be clearly seen. In addition, it has also been found that $t_{\mathrm{c}}, t_{\mathrm{s}}$, and $E_{\mathrm{s}}$ in this case follows eq. (6) closely (data not shown). Another example is shown in Figure 5 for a more general non-KAM potential:

$V_{\mathrm{D}}(\theta)= \begin{cases}1-2(\theta / \pi)^{2}, & \text { for }-\pi \leqslant \theta<0, \\ 2(\theta / \pi-1)^{2}-1, & \text { for } \quad 0 \leqslant \theta<\pi .\end{cases}$

This is a piecewise quadratic potential "randomly" designed with the only requirement of symmetry $V_{\mathrm{D}}(\theta)=-V_{\mathrm{D}}(\theta+\pi)$. It has been seen that the superballistic spreading occurs again with the same characteristics.

It is interesting to notice that the symmetry of potential $V(\theta)=-V(\theta+\pi)$ plays an important role for both the superballistic spreading observed in this work and the exponential spreading observed in ref. [6] with $V_{\mathrm{S}}(\theta)=\cos (\theta)$. Indeed, we find that if we replace $V_{\mathrm{S}}$ with $V(\theta)=\cos (m \theta)$ where $m$ is instead an even integer, which is a KAM-type potential but
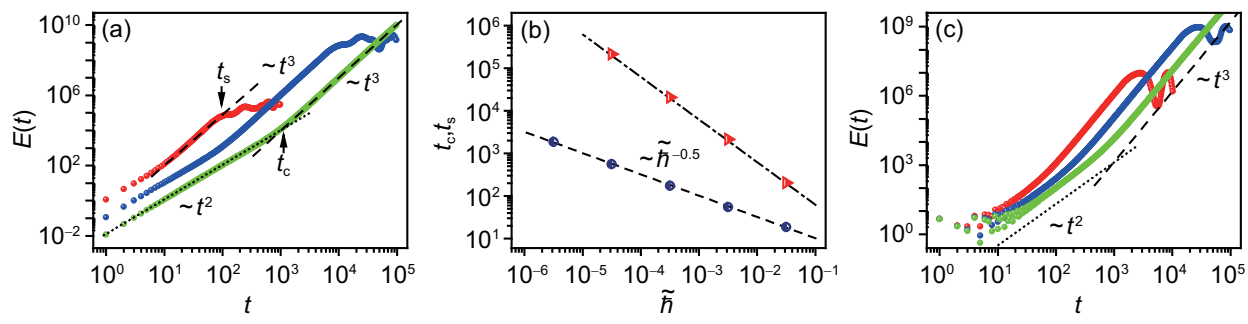

Figure 4 (Color online) (a) Time dependence of the energy of the QDKR with potential $V_{\mathrm{A}}$ at $\hbar=2 \pi+\tilde{\hbar}$. The red, blue, and green solid bullets (from left to right) are for, respectively, $\tilde{\hbar}=0.1,10^{-3}$, and $10^{-5}$. The dotted and the dashed lines indicate the time scaling $\sim t^{2}$ and $\sim t^{3}$, respectively. Two time scales, $t_{\mathrm{c}}$ and $t_{\mathrm{s}}$, that characterize the $t^{2}-t^{3}$ crossover and the suppressing of $\sim t^{3}$ increasing, are determined by best fitting the corresponding increasing stage and extrapolating the best fitting lines (examples are shown here). (b) The results of the measured $t_{\mathrm{c}}$ and $t_{\mathrm{s}}$ for various $\tilde{\hbar}$ are presented by circles and triangles, respectively, as a comparison with the predicted scaling $t_{\mathrm{c}} \sim 1 /\left(K \tilde{\hbar}^{0.5}\right)$ (dashed line) and $t_{\mathrm{s}} \approx \pi^{2} \hbar /(2 K \tilde{\hbar})$ (dot-dashed line). It can be seen that both are in good agreement; (c) the results of $E(t)$ for $\hbar=2 \pi / 3+\tilde{\hbar}$ instead. From left to right, the red, blue, and green bullets are for $\tilde{\hbar}=10^{-2}, 10^{-3}$, and $10^{-4}$, respectively.
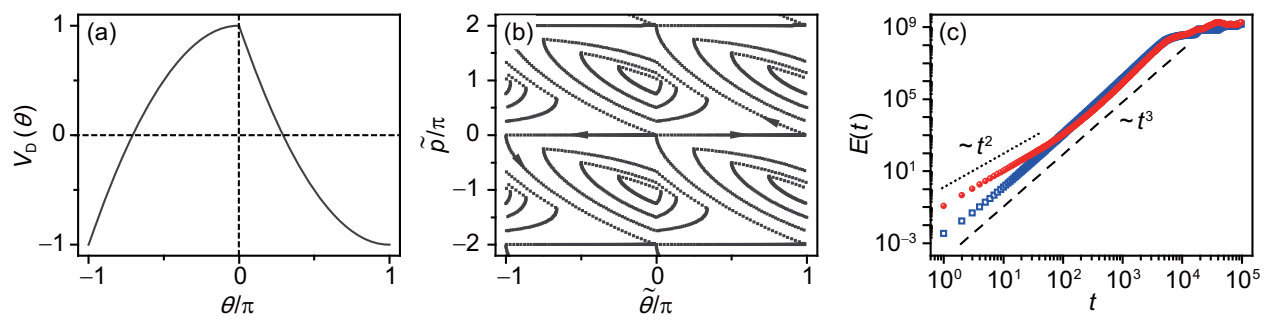

Figure 5 (Color online) The superballistic wavepacket spreading occurs in the QDKR with a more general non-KAM potential, $V_{\mathrm{D}}$, given by eq. (20). (a) The potential $V_{\mathrm{D}}$ has the symmetry $V_{\mathrm{D}}(\theta)=-V_{\mathrm{D}}(\theta+\pi)$; (b) the phase portrait of the corresponding pseudoclassical system; (c) time dependence of the energy of the QDKR (red solid bullets) and its pseudoclassical system (blue open squares). For (b) and (c), the system parameters are $K=5, \hbar=2 \pi+\tilde{\hbar}$ and $\tilde{\hbar}=10^{-3}$. 
loses the symmetry of $V(\theta)=-V(\theta+\pi)$, then the exponential spreading no longer happens; rather, it is replaced by the ballistic spreading. For $V(\theta)=\cos (m \theta)$ with even $m$, we find that the phase space structure of the corresponding pseudoclassical system at $\hbar \approx 2 \pi$ is similar to Figure 3(c): The key structure along the $\tilde{\theta}$ axis in the case of $V_{\mathrm{S}}$, i.e., the stable and unstable manifolds of the hyperbolic fixed points $(\tilde{\theta}, \tilde{p})=(-\pi, 0)$ and $(0,0)$ that are essential to the exponential spreading [6], vanishes. Therefore, the role the symmetry $V(\theta)=-V(\theta+\pi)$ plays, is to create a special structure along the $\tilde{\theta}$ axis which in turn is taken full advantage by the initial conditions around the $\tilde{\theta}$ axis (see the discussion at the end of sect. 4). In our non-KAM QDKR, the profound difference in quantum dynamics lies in the phase space structure (of the pseudoclassical system) along the $\tilde{\theta}$ axis: Due to the nonKAM nature of the potential, the hyperbolicity of the fixed points is destroyed and the phase points on the $\tilde{\theta}$ axis do not move exponentially.

To summarize, we have shown that the superballistic wavepacket spreading can happen in a class of QDKR systems provides, (i) $\hbar \approx 2 \pi M / N$ where $M$ and $N$ are odd coprime integers; (ii) the system is non-KAM; and (iii) the potential has the symmetry $V(\theta)=-V(\theta+\pi)$. The wavepacket spreading will become exponential if the system is KAMtype or ballistic if the potential loses the required symmetry. Our study evidences the effectiveness of the pseudoclassical limit theory and the rich dynamics of the QKR. An interesting question is whether the hyperdiffusive wavepacket spreading $(\gamma>3)$ is possible in the QKR and its variants, which we leave for future studies.

This work was supported by the National Natural Science Foundation of China (Grants Nos. 11275159, 11535011 and 11335006).
1 R. Ketzmerick, K. Kruse, S. Kraut, and T. Geisel, Phys. Rev. Lett. 79, 1959 (1997).

2 I. Guarneri, Europhys. Lett. 10, 95 (1989).

3 I. Guarneri, Europhys. Lett. 21, 729 (1993).

4 L. Hufnagel, R. Ketzmerick, T. Kottos, and T. Geisel, Phys. Rev. E 64, 012301 (2001)

5 Z. J. Zhang, P. Q. Tong, J. B. Gong, and W. B. Li, Phys. Rev. Lett. 108, 070603 (2012).

6 J. Wang, I. Guarneri, G. Casati, and J. B. Gong, Phys. Rev. Lett. 107, 234104 (2011).

7 G. Casati, B. V. Chirikov, F. M. Izrailev, and J. Ford, in Stochastic behavior in classical and quantum Hamiltonian systems (Lecture Notes in Physics Vol.93) (Springer, Berlin, 1979), pp. 334-352.

8 F. L. Moore, J. C. Robinson, C. F. Bharucha, B. Sundaram, and M. G. Raizen, Phys. Rev. Lett. 75, 4598 (1995).

9 J. Chabe, G. Lemarie, B. Gremaud, D. Delande, P. Szriftgiser, and J. C. Garreau, Phys. Rev. Lett. 101, 255702 (2008).

10 H. L. Wang, J. Wang, I. Guarneri, G. Casati, and J. B. Gong, Phys. Rev. E 88, 052919 (2013).

11 S. Fishman, I. Guarneri, and L. Rebuzzini, Phys. Rev. Lett. 89, 084101 (2002).

12 F. M. Izrailev, and D. L. Shepelyanskii, Theor. Math. Phys. 43, 553 (1980).

13 I. Dana, E. Eisenberg, and N. Shnerb, Phys. Rev. E 54, 5948 (1996).

14 L. Chierchia, and J. N. Mather, Scholarpedia 5, 2123 (2010).

15 P. X. Wang, J. X. Wang, Y. K. Huo, W. Scheid, and H. Hora, Chin. Sci. Bull. 57, 1494 (2012).

16 A. Shapira, L. Naor, and A. Arie, Sci. Bull. 60, 1403 (2015).

17 P. F. Sui, and Z. H. Dai, Sci. China-Phys. Mech. Astron. 58, 052002 (2015).

18 B. Chirikov, and D. Shepelyansky, Scholarpedia 3, 3550 (2008).

19 W. Li, S. G. Chen, and G. R. Wang, Phys. Lett. A 281, 334 (2001).

20 P. H. Jones, M. M. Stocklin, G. Hur, and T. S. Monteiro, Phys. Rev. Lett. 93, 223002 (2004).

21 J. Wang, T. S. Monteiro, S. Fishman, J. P. Keating, and R. Schubert, Phys. Rev. Lett. 99, 234101 (2007).

22 W. Lawton, A. S. Mouritzen, J. Wang, and J. B. Gong, J. Math. Phys. 50, 032103 (2009).

23 J. Wang, and J. B. Gong, Phys. Rev. A 77, 031405(R) (2008). 